\title{
Tuning the Optical Absorption of Anatase Thin Films Across the Visible-To-Near-Infrared Spectral Region
}

\author{
Pasquale Orgiani®, ${ }^{1,2,{ }^{*}}$ Andrea Perucchi, ${ }^{3}$ Daniel Knez@ ${ }^{1}$ Regina Ciancio, ${ }^{1}$ Chiara Bigi $\odot, 1,4$ \\ Sandeep Kumar Chaluvadi, ${ }^{1}$ Jun Fujii, ${ }^{1}$ Ivana Vobornik, ${ }^{1}$ Giancarlo Panaccione $\odot,{ }^{1}$ Giorgio Rossi, ${ }^{1,4}$ \\ Stefano Lupi, ${ }^{1,5}$ and Paola Di Pietro ${ }^{3}$ \\ ${ }^{1}$ CNR-IOM TASC Laboratory, Trieste 34139, Italy \\ ${ }^{2}$ CNR-SPIN, UOS Salerno, Fisciano, SA 84084, Italy \\ ${ }^{3}$ Elettra Sincrotrone Trieste S.C.p.A., Trieste 34139, Italy \\ ${ }^{4}$ Department of Physics, University of Milano, Milano 20133, Italy \\ ${ }^{5}$ Department of Physics, University of Roma La Sapienza, Roma 00185, Italy
}

(Received 25 October 2019; revised manuscript received 6 March 2020; accepted 19 March 2020; published 6 April 2020)

\begin{abstract}
The electronic properties of anatase titanium dioxide $\left(\mathrm{TiO}_{2}\right)$ thin films epitaxially grown on $\mathrm{LaAlO}_{3}$ substrates are investigated by synchrotron-x-ray spectroscopy [x-ray absorption spectroscopy (XAS), xray photoemission spectroscopy (XPS), and angle-resolved photoemission spectroscopy (ARPES)] and infrared spectroscopy. The $\mathrm{Ti}^{3+}$ fraction in $\mathrm{TiO}_{2-x}$ is varied either by changing the oxygen pressure during deposition or by postgrowth annealing in ultrahigh vacuum (UHV). Structural investigation of the $\mathrm{TiO}_{2}$ thin films provides evidence of highly uniform crystallographic order in both as-grown and in situ UHVannealed samples. The increased amount of $\mathrm{Ti}^{3+}$ as a consequence of UHV annealing is calibrated by in situ XPS and XAS analysis. The as-grown $\mathrm{TiO}_{2}$ samples, with a low $\mathrm{Ti}^{3+}$ concentration, show distinct electronic properties with respect to the annealed films, namely, absorption in the midinfrared (MIR) region correlated with polaron formation, and another peak in the visible range at $1.6 \mathrm{eV}$ correlated with the presence of localized defect states (DSs). With the increasing level of $\mathrm{Ti}^{3+}$ induced by the postannealing process, the MIR peak disappears, while the DS peak is redshifted to the near-infrared region at about 1.0 $\mathrm{eV}$. These results indicate the possibility of tailoring the optical absorption of anatase $\mathrm{TiO}_{2}$ films from the visible to the near-infrared region.
\end{abstract}

DOI: 10.1103/PhysRevApplied.13.044011

\section{INTRODUCTION}

The surface properties of titanium dioxide $\left(\mathrm{TiO}_{2}\right)[1-4]$ make this system a very widely studied oxide for possible applications in photocatalysis [5,6] and many other fields [7-10]. An impressive number of real devices based on the photocatalytic properties of $\mathrm{TiO}_{2}$ are nowadays available, such as devices for photoinduced water photolysis, photocatalytic devices, photocatalytic self-cleaning $\mathrm{TiO}_{2}$-coated materials, devices using the photocatalytic antibacterial effect of $\mathrm{TiO}_{2}$ films, devices using photoinduced hydrophilicity, and many others [5]. Anatase $\mathrm{TiO}_{2}$, which is considered potentially most relevant for energyrelated applications from among the three different $\mathrm{TiO}_{2}$ polymorphs, is an insulator with a band gap of $3.2 \mathrm{eV}$ $[11,12]$. It therefore shows very efficient photoactivity under UV irradiation, but this accounts for only $5 \%$ of the solar spectrum, limiting its use in wide-scale applications. However, it has been recently shown that the presence of

\footnotetext{
*pasquale.orgiani@spin.cnr.it
}

$\mathrm{Ti}^{3+}$ impurities extends the photoresponse of a polycrystalline mixture of rutile and anatase $\mathrm{TiO}_{2-x}$ from the UV to the visible-light region. The appearance of band-gap states may indeed lead to high visible-light photocatalytic activity $[13,14]$.

Here we report on the evolution of the electronic properties of epitaxial anatase $\mathrm{TiO}_{2-x}$ thin films that show different fractions of oxygen defects $(x)$, as studied by a combination of x-ray, optical, and photoelectron spectroscopy. The variable concentration of oxygen defects arising from the growth and postgrowth conditions is reflected in the results of core-level x-ray spectroscopy. Combined analysis of data from angle-resolved photoemission spectroscopy (ARPES) and infrared (IR) spectroscopy shows the development of defect-related bands and an IR absorption peak that shifts in energy from 1.6 to $1 \mathrm{eV}$. The results indicate the possibility of tuning the optical absorption of anatase $\mathrm{TiO}_{2}$ thin films in the visible and near-infrared spectral region, i.e., of exploiting a substantial part of the solar spectrum (e.g., $42 \%-43 \%$ of it falls in the visible range, while the remaining part is mostly 
in the infrared region), therefore opening up the possibility of $\mathrm{TiO}_{2}$-based optical devices with tailored electronic properties.

\section{EXPERIMENTAL PROCEDURES}

\section{A. Growth and structural characterization}

Anatase $\mathrm{TiO}_{2-x}$ thin films are grown by pulsed laser deposition using a $\mathrm{KrF}$ excimer pulsed laser source at the in situ NFFA facility, which integrates the APE beamlines at IOM-CNR at Elettra in Trieste [15,16]. A typical energy density of about $2 \mathrm{~J} / \mathrm{cm}^{2}$ and a laser repetition rate of $1 \mathrm{~Hz}$ are used. The growth temperature is set to $700^{\circ} \mathrm{C}$, and the target-substrate distance is about $5 \mathrm{~cm}$. A stoichiometric single-crystalline rutile $\mathrm{TiO}_{2}$ target is used for the deposition process. Epitaxial anatase $\mathrm{TiO}_{2-x}$ thin films are grown on (001)-oriented $\mathrm{LaAlO}_{3}$ (LAO) under a wide range of oxygen background pressures (i.e., from $10^{-5}$ up to $1 \mathrm{mbar}$ ). For the present study, the oxygen pressure is fixed at $3 \times 10^{-4}$ and $1 \times 10^{-4}$ mbar (hereafter called asgrown samples no. 1 and no. 2). Such values have been proven to induce a very small amount of oxygen vacancies in $\mathrm{TiO}_{2}$ thin films [17]. The annealing process is performed by letting the sample remain at the deposition temperature (i.e., $700^{\circ} \mathrm{C}$ ) in UHV (i.e., at a base pressure less than $10^{-7} \mathrm{mbar}$ ); this sample is referred to hereafter as the annealed film. The crystallographic properties of the films are probed ex situ by means of x-ray diffraction (XRD) using a four-circle diffractometer with a $\mathrm{Cu} K_{\alpha}$ radiation source. The film thickness and surface roughness are probed by low-angle $\mathrm{x}$-ray reflectivity (XRR). XRR and symmetrical $\theta-2 \theta$ measurements of the as-grown sample no. 1 and the annealed sample are shown in Fig. 1.

Numerical simulations of the low-angle XRR data (i.e., the blue curve in the upper panel of Fig. 1), performed by means of the IMD package in the XOP software [18,19], fully match the design value of $30 \mathrm{~nm}$. XRR oscillations are recorded up to $2 \theta$ values of $4^{\circ}$, while, above this angle, the oscillations fall below the experimental sensitivity of the x-ray diffractometer [17,20,21]. In situ scanningtunneling-microscopy (STM) experiments are performed with an atomic-resolution UHV apparatus, immediately after the growth of the samples. The STM topography shows good surface quality, with an overall root-meansquare value of surface roughness of about $0.2 \mathrm{~nm}$, thus implying a very low surface roughness of the grown samples, consistently with previous studies [17].

The symmetrical $\theta-2 \theta$ XRD scans contain only anatase diffraction peaks, indicating that the samples are singlephase and well oriented, with no traces of secondary phases or other crystallographic orientations. Precise determination of the out-of-plane lattice parameters is done after alignment of the (002) diffraction peaks of the LAO substrate [22]. The out-of-plane lattice parameter of the as-grown sample no. 1, calculated from the symmetric
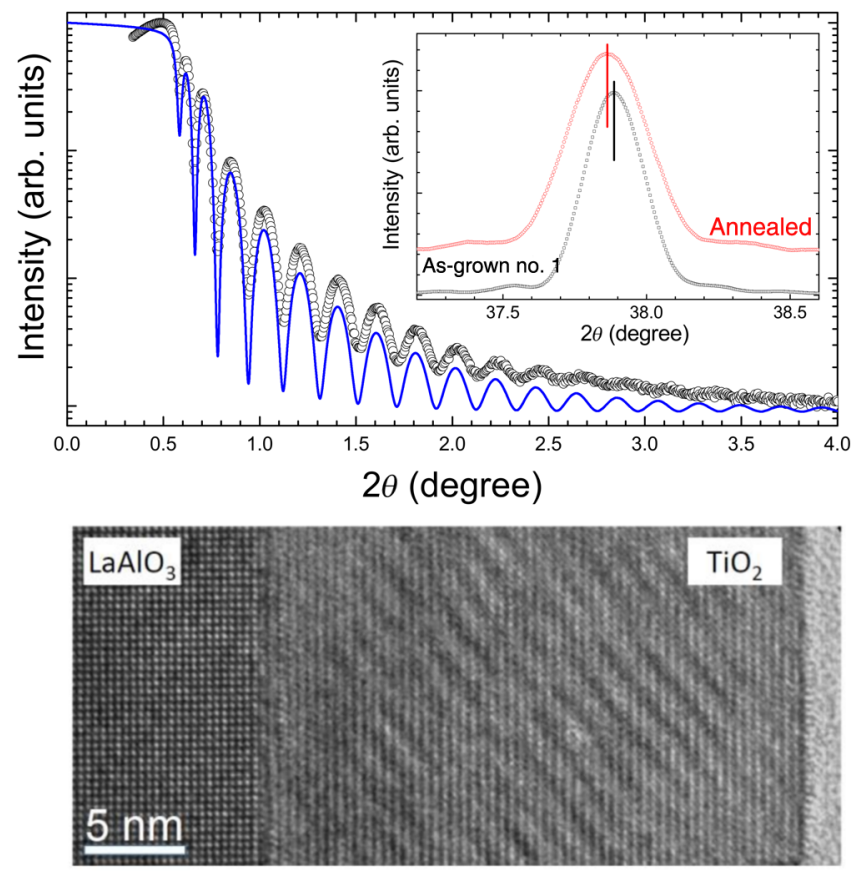

FIG. 1. Upper panel: low-angle XRR of 30-nm-thick $\mathrm{TiO}_{2-x}$ film grown on (001) LAO (the simulated XRR curve is also included as a continuous blue line). The inset shows symmetrical $\theta-2 \theta$ scans of the as-grown sample no. 1 (black) and the annealed $\mathrm{TiO}_{2-x}$ film (red). Lower panel: HRTEM image of an annealed $\mathrm{TiO}_{2-x}$ film taken along the [010] zone axis.

(004) Bragg reflections and shown in the inset of Fig. 1, is $0.947 \pm 0.001 \mathrm{~nm}$, in good agreement with the $c$-axis parameter of relaxed anatase $\mathrm{TiO}_{2}$. This is expected due to the very low lattice mismatch between the LAO substrate and the anatase $\mathrm{TiO}_{2}(0.1 \%$, i.e., a strainless condition). However, as a consequence of the loss of oxygen in the annealing process, and similarly to what occurs in many other perovskite oxide systems [23-26], the out-of-plane lattice parameter increases to $0.949 \pm 0.001 \mathrm{~nm}$.

The atomic structure of the $\mathrm{TiO}_{2}$ films is investigated by high-resolution transmission electron microscopy (HRTEM). Similarly to previously investigated as-grown samples [17,27,28], HRTEM reveals the presence of a modulated structure all through the film thickness in the case of UHV-annealed $\mathrm{TiO}_{2}$ films also; this is tentatively associated with the occurrence of oxygen vacancies, selforganizing into regular arrays of planar defects. Importantly, no structural differences can be seen between the near-interface region and the bulk of the film, as well as no traces of spurious phases or segregation of crystalline phases other than anatase, consistently with the XRD results.

\section{B. Characterization by core-level spectroscopy}

Soft-x-ray synchrotron radiation spectra [obtained by $\mathrm{x}$-ray absorption spectroscopy (XAS) and x-ray 


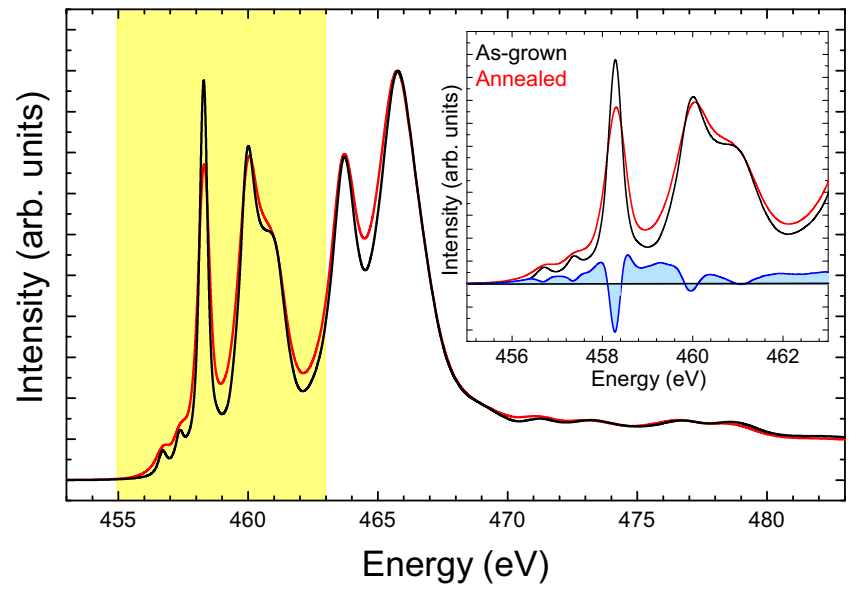

FIG. 2. XAS spectra at the Ti $L$ edge of the as-grown sample no. 1 (black) and the annealed (red) $\mathrm{TiO}_{2}$ film. The inset shows an enlargement of the $\mathrm{Ti} L_{3}$-edge spectral region (the yellow box in the main panel) along with the difference curve (shown in blue, and defined as the curve for the annealed sample minus that for the as-grown sample no. 1).

photoemission spectroscopy (XPS)] are measured on samples transferred in situ directly after growth, under UHV conditions (base pressure less than $2 \times 10^{-10} \mathrm{mbar}$ ), to the two APE end-stations $[15,16]$. The energy resolution for both the XPS and the XAS experiments is about $100 \mathrm{meV}$. The XAS experiments are performed in the total-electronyield mode, and the drain current from a highly transparent mesh, upstream of the sample, was used to normalize the signal with the incident photon flux. XAS spectra of the Ti $L_{2,3}$ absorption edge of as-grown and annealed $\mathrm{TiO}_{2-x}$ films are shown in Fig. 2.

The spectrum is divided into four main peaks: the first two peaks (at about 458 and $460 \mathrm{eV}$ ) correspond to the $L_{3}$ absorption transitions, and the last two peaks (at about 463 and $465 \mathrm{eV}$ ) correspond to the $L_{2}$ transitions. In particular, the second peak of the $L_{3}$ transitions (i.e., around $460 \mathrm{eV}$ ) shows the characteristic splitting of the $e_{g}$ levels of anatase $\mathrm{TiO}_{2}$ for both samples. The effect of the annealing process is clearly visible in the preedge region, as well as between the two peaks corresponding to $L_{3}$ at about $459 \mathrm{eV}$, where Ti ions in the $3+$ valence state dominate $[29,30]$. As expected, the increased amount of $\mathrm{Ti}^{3+}$ in the annealed sample can be traced by the increased intensity of the preedge region and the valley region between the $L_{3}$ absorption peaks in the XAS spectra, as revealed by the difference curve (defined as the curve for the annealed sample minus that for the as-grown sample no. 1) shown in the inset of Fig. 2.

The chemical composition of the $\mathrm{TiO}_{2}$ thin films is quantitatively explored by measuring the intensities of core-level photoemission spectra (Fig. 3). As expected, the Ti $2 p$ XPS spectra display two main peaks, at binding energies of 459.4 and $465.1 \mathrm{eV}$, corresponding to $\mathrm{Ti}_{2 p}^{3 / 2}$
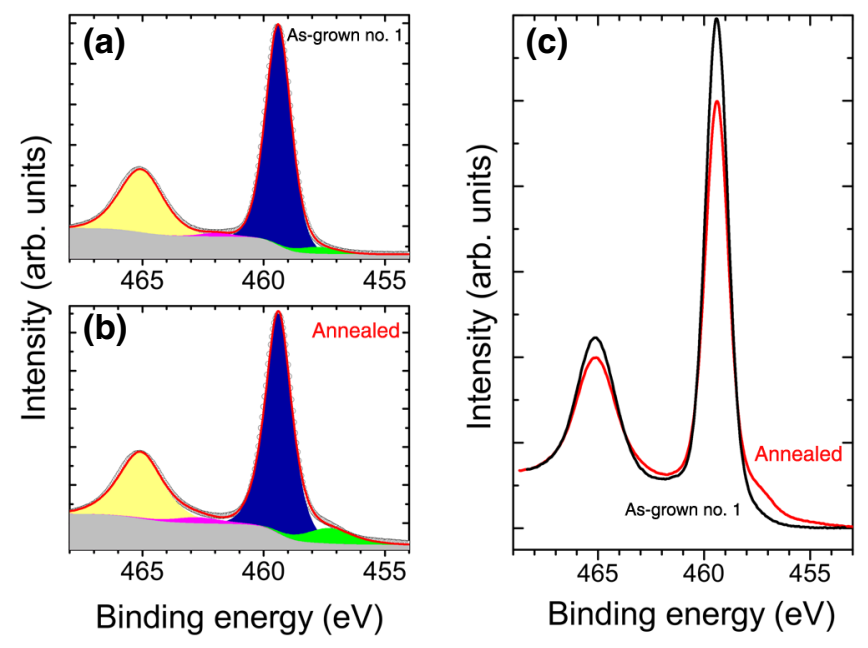

FIG. 3. Ti $2 p$ XPS peaks, showing the main environment $\left(\mathrm{Ti}^{4+}\right.$, blue and yellow) and the suboxide environment $\left(\mathrm{Ti}^{3+}\right.$, green and magenta), of the as-grown sample no. 1 (a) and the annealed $\mathrm{TiO}_{2-x}$ sample (b). The Ti $2 p$ XPS peaks also overlap in panel (c). The spectra are measured with a photon energy of $900 \mathrm{eV}$.

and $\mathrm{Ti}_{2 p}^{1 / 2}$, respectively, in the $4+$ valence state, flanked by several satellites (not shown in the graph) as described in the literature [17,31-33]. However, the presence of a small amount of atoms in the $\mathrm{Ti}^{3+}$ configuration, usually related to oxygen vacancies, is revealed by a small shoulder on the lower-binding-energy side of the main Ti $2 p$ peak [31-33].

The amount of $\mathrm{Ti}^{3+}$ can therefore be calculated from the ratio of the area of the $\left[\mathrm{Ti}^{3+}\right]_{2 p}^{3 / 2}$ peak to the area of the $\left(\left[\mathrm{Ti}^{4+}\right]_{2 p}^{3 / 2}+\left[\mathrm{Ti}^{3+}\right]_{2 p}^{3 / 2}\right)$ peaks. Since the formation of oxygen vacancies is equivalent to the addition of two electrons per $\mathrm{Ti}$ ion (i.e., two $\mathrm{Ti}^{4+}$ therefore become $\mathrm{Ti}^{3+}$ ), the evaluation of the $\mathrm{Ti}^{3+}$ abundance provides a direct measure of the oxygen off-stoichiometry in the $\mathrm{TiO}_{2-x}$ films (i.e., $x=1 / 2 \times$ amount of $\mathrm{Ti}^{3+}$ ). Fitting of these XPS peaks is performed after the removal of a Shirley background assuming a statistical intensity ratio between $\mathrm{Ti}_{2 p}^{3 / 2}$ and $\mathrm{Ti}_{2 p}^{1 / 2}$ of 0.5 for each valence state $(3+$ and $4+$ ). A Gaussian-Lorentzian fitting function, with fixed parameters, was used to fit all peaks presented in both Fig. 3(a) and Fig. 3(b).

As already reported, the amount of $\mathrm{Ti}^{3+}$ in the as-grown films differs for samples grown at different oxygen pressures during deposition, although in a nonmonotonic way [17]. The overall amount of $\mathrm{Ti}^{3+}$ in the as-grown $\mathrm{TiO}_{2-x}$ films is relatively low (i.e., $\mathrm{Ti}^{3+}=2 \%$ and $4 \%$ for the asgrown samples no. 1 and no. 2 , respectively). In contrast, even a mild postannealing process of the films provides a much higher concentration of oxygen vacancies (i.e., $\left.\mathrm{Ti}^{3+}=11 \%\right)$. Such results confirm that the UHV annealing process promotes loss of oxygen from the $\mathrm{TiO}_{2-x}$ thin films. 


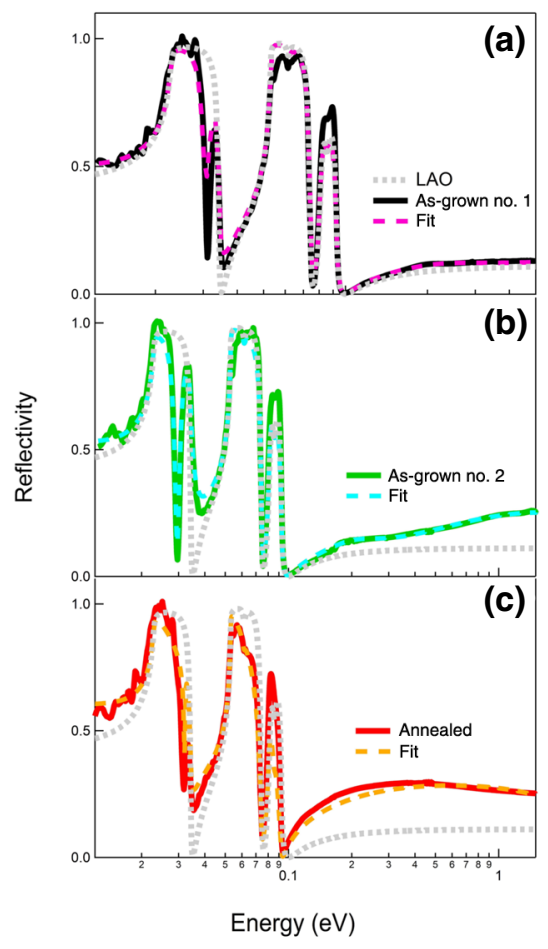

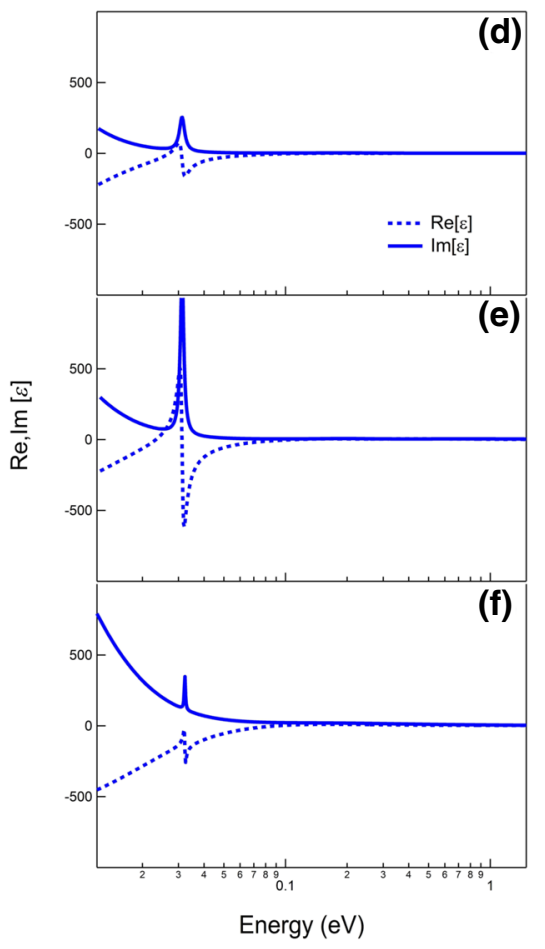

FIG. 4. Left: reflectivity data for the three $\mathrm{TiO}_{2-x}$ samples investigated over the whole IR range, i.e., the as-grown sample no. 1 (a), the as-grown sample no. 2 (b), and the annealed sample (c). Each panel also contains reflectivity data for the bare LAO substrate and a Drude-Lorentz fit to the sample reflectivity. Right: real and imaginary parts of the dielectric function calculated from the fitting parameters for the same samples, i.e., the as-grown sample no. 1 (d), the as-grown sample no. 2 (e), and the annealed sample (f).

\section{Infrared spectroscopy and ARPES}

Measurements of the reflectivity of the samples that are analyzed as described in the previous sections (i.e., the two as-grown samples and one annealed sample) are performed at the SISSI-Material Science beamline at Elettra [34]. The whole IR range is covered by means of a Bruker $70 \mathrm{v}$ interferometer, using a gold mirror as a reference and various beam splitters, detectors, and thermal sources. In particular, in the lowest-frequency region, we take advantage of the high brilliance of the infrared synchrotron light delivered by Elettra. A He-cooled bolometer is used as a detector in the far IR, while for the mid-IR and visible ranges, a conventional source and $\mathrm{MCT}$ and Si diodes are used.

Figure 4 shows reflectivity data (left panel) for and the relative dielectric function (right panel) of the three samples investigated and the LAO substrate, over the whole IR range. In the far-IR range, three main peaks are clearly distinguishable and are ascribed to the substrate. However, the phonon peak at about $0.03 \mathrm{eV}$ is related to an infrared-active phonon of $\mathrm{TiO}_{2}$, as identified in the literature [35]. Such a phonon appears to be more screened in the most metallic case (i.e., the annealed sample), when the reflectivity is also higher.

The data reported in Fig. 4(a)-Fig. 4(c) (solid lines) are fitted by a Drude-Lorentz model, which provides the normal-incidence reflectivity of a thin film grown on a substrate when the optical properties [namely $n(\omega)$ and $k(\omega)$ ] are known. In particular, the fitting procedure takes into account the thickness of both the film and the substrate to calculate the best reflectivity by optimizing the
Drude-Lorentz parameters. Once the best-fitting DrudeLorentz parameters are obtained, the related dielectric function and hence the optical conductivity can be analytically calculated. The results of the fit to the data are shown in Fig. 4(d)-Fig. 4(f).

To better highlight the Drude term and the midinfrared components, the Lorentzian parameters related to the IRactive phonon of anatase $\mathrm{TiO}_{2}$ (the peak at $0.03 \mathrm{eV}$ in Fig. 4(a)-Fig. 4(c), dashed lines) are removed to calculate the phonon-subtracted optical conductivity (shown in Fig. 5). In particular, the Drude term, due to the contribution of free carriers to the conductivity, can be recognized in the far-IR range. A second component can be clearly ascertained in the mid-IR (MIR) band for the as-grown samples only. This component, centered at 0.21 and at $0.20 \mathrm{eV}$ for the as-grown samples no. 1 and no. 2 , respectively, is tentatively attributed to polaronic absorption. As a matter of fact, the formation of polarons has been widely predicted and investigated in $\mathrm{TiO}_{2-x}$ [36-43], as well as in other oxide materials [44-49]. Indeed, in the present case, the controlled oxygen pressure during growth determines the presence of oxygen vacancies, which distort the lattice and promote polaron formation. In this scenario, the MIR band is related to the incoherent part of the electron motion and represents a good estimate of the energy for photoionizing the polaron particle, i.e., the process of making the self-trapped carrier free by the absorption of a photon $[36,37,39,40,50]$. The third optical component falls into the near-IR and visible band and represents the interband transition related to the absorption of an electronic defect state (DS) previously associated 

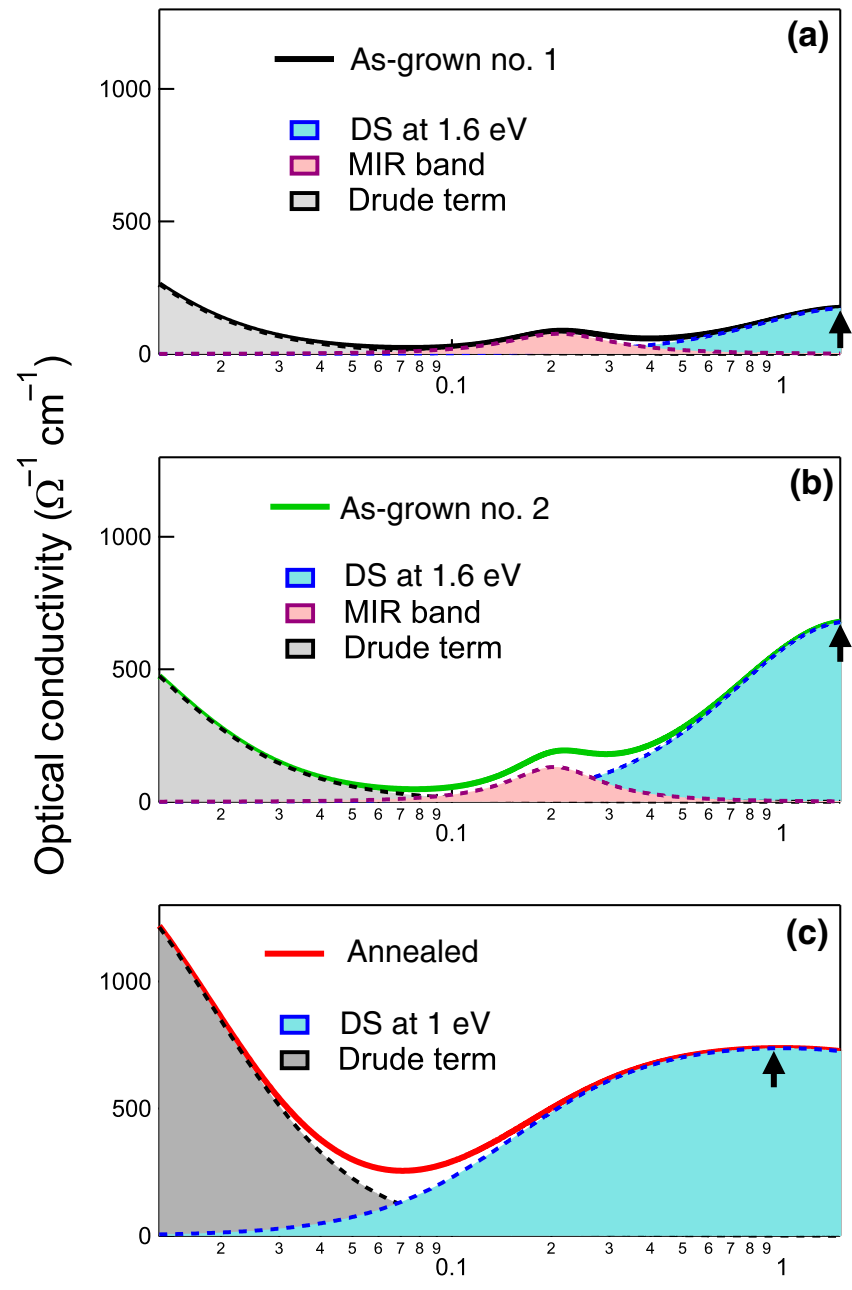

Energy $(\mathrm{eV})$

FIG. 5. Optical conductivity of the three samples under investigation, after subtraction of the $\mathrm{TiO}_{2}$ phonon at about $0.03 \mathrm{eV}$.

with oxygen vacancies in similar compounds [51,52]. A comparison of the three optical conductivities highlights a transfer of spectral weight from the visible range in the as-grown samples to the near IR in the annealed sample (Fig. 6).

The Drude term becomes increasingly intense from the most insulating case (i.e., the as-grown sample no. 1) to the most metallic case (i.e., the annealed sample). The MIR band, which is present in the as-grown cases alone, is slightly redshifted in the case of the as-grown sample no. 2, for which the concentration of oxygen vacancies is lower. Contrariwise, in the annealed case, the MIR band disappears and merges into the more intense and broader Drude term. On the other hand, the DS-peak intensity increases as the oxygen-vacancy concentration increases. Moreover, the DS central frequency, which is found to be substantially constant at $1.6 \mathrm{eV}$ in both of the as-grown

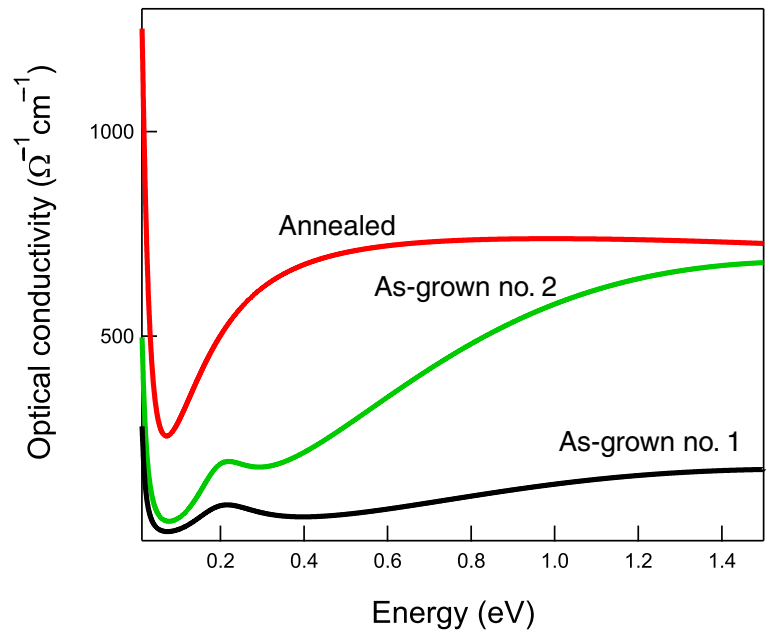

FIG. 6. Fitted Drude, MIR, and DS-band contributions to the optical conductivity of the three samples under investigation, after subtraction of the $\mathrm{TiO}_{2}$ phonon at about $0.03 \mathrm{eV}$.

samples, is noticeably redshifted to $1.0 \mathrm{eV}$ in the annealed sample.

It has been demonstrated [53] that the volume carrier density $n_{3 \mathrm{D}}$ can be derived from the Drude spectral weight $W_{\text {Drude }}$ (namely, the area underneath the Drude term of the optical conductivity, shown as the gray area in Fig. 5) from the formula

$$
n_{3 \mathrm{D}}=\frac{m^{*}}{4 \pi e^{2}} W_{\text {Drude }}
$$

where $m^{*}$ is the effective mass, which can be experimentally determined from ARPES experiments. ARPES spectra are acquired on in-situ-transferred $\mathrm{TiO}_{2}$ thin films by a Scienta D30 spectrometer using linearly polarized radiation of energy $46 \mathrm{eV}$ and with the sample at a temperature of $77 \mathrm{~K}$. The energy resolution for the ARPES experiments is about $40 \mathrm{meV}$. The ARPES spectrum [Fig. 7(b)] shows an outer parabola and a subband at a relative separation of approximately $100 \mathrm{meV}$. This subband is known to arise from the confinement potential at the surface and suggests that the metallic state is a two-dimensional electron gas (2DEG) [54-56]. The parabolic dispersive bands allow one to derive the effective mass of free carriers $m^{*}$, as this is reflected in their curvature through the formula

$$
m^{*}=\hbar^{2} \frac{\partial^{2} E(k)}{\partial^{2} k}
$$

From the fitting parameters for the parabolic state [the red dotted line superimposed on the ARPES data in Fig. 7(b)], we obtain an effective mass $m^{*}$ equal to $2.2 m_{e}$, consistent with data reported for similar oxide systems $[57,58]$. The volume carrier density $n_{3 \mathrm{D}}$ derived from $W_{\text {Drude }}$ is therefore calculated and is reported in Fig. 7(a) (black filled 


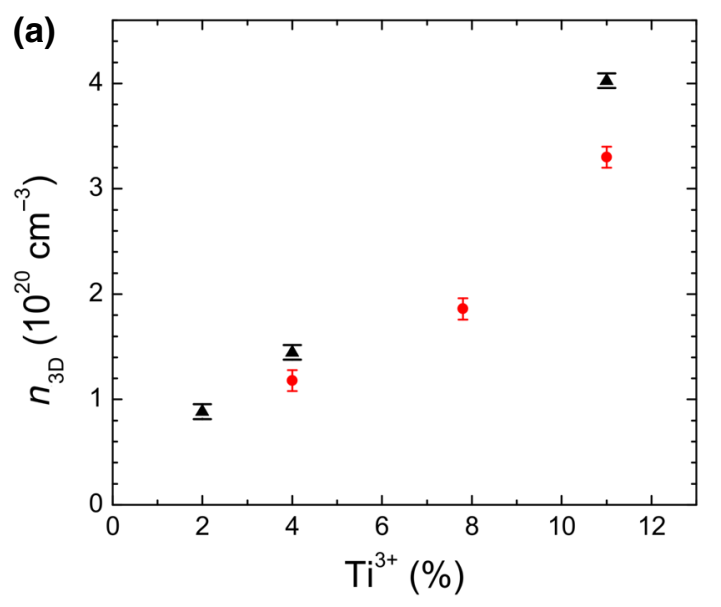

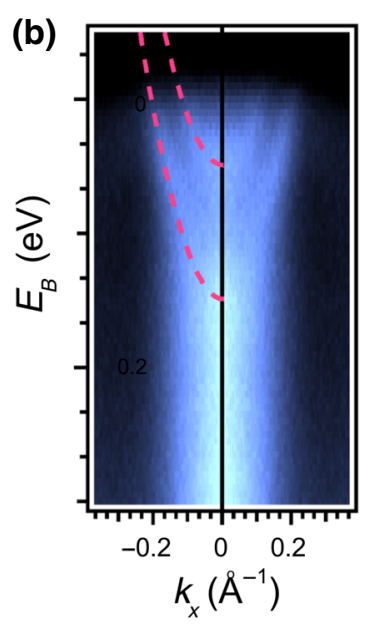

FIG. 7. (a) Carrier densities associated with the Drude term, $n_{D}$ (black filled triangles), and those derived from the ARPES measurements, $n_{\text {ARPES }}$ (red filled circles), as a function of the $\mathrm{Ti}^{3+}$ content. (b) ARPES data at the center of the second Brillouin zone of the annealed sample. The red dotted lines result from the data fitting and are superimposed on the spectra as a guide to the eye. triangles), in which one can see how the electron density increases linearly with the $\mathrm{Ti}^{3+}$ content.

The carrier density can be independently extracted from the ARPES data as well. From our previous study, the chemical composition of $\mathrm{TiO}_{2}$ thin films is found to be homogeneous throughout the film thickness [17]. Therefore the distribution of the oxygen vacancies (i.e., of the excess carriers) in the samples is uniform throughout the material (i.e., in the bulk and surface). At the surface (i.e., in the probing depth accessible by ARPES), the excess electrons associated with those vacancies manifest themselves as a 2DEG state [56]. In this respect, Luttinger's theorem [59] relates the free-carrier density to the area of the Fermi surface. In particular, in the case of a spindegenerate two-dimensional state, the surface carrier density is given by the formula $k_{F}^{2} / 2 \pi$, where $k_{F}^{2}$ is the Fermi momentum of the parabolic states. As also described in the literature $[39,41,43,54,57]$, the three-dimensional density of states can therefore be estimated as $n_{3 \mathrm{D}}^{\mathrm{ARES}}=n_{2 \mathrm{D}}^{3 / 2}$ and, finally, compared with the three-dimensional density of states extracted from the Drude term. The consistency between the carrier densities estimated with these two independent methods indicates that the primary source of free carriers in the otherwise insulating anatase samples is the oxygen vacancies, which are homogeneously distributed throughout the sample thickness. Furthermore, while in the bulk of the material they induce a number of electronic states that can be probed by IR spectroscopy, at the surface the very same oxygen vacancies produce a highly metallic 2DEG.

\section{CONCLUSIONS}

The structural, electronic, and optical properties of anatase $\mathrm{TiO}_{2-x}$ thin films are investigated as a function of oxygen-vacancy concentration, which translates into charge-carrier density. As-grown $\mathrm{TiO}_{2-x}$ samples grown under a controlled oxygen background pressure are unique in displaying a polaronic phenomenology. They show a low concentration of oxygen vacancies (i.e., 3\%), and this generates localized electron states. In contrast, oxygen loss leads to complex changes in the optical and electronic properties that cannot be explained simply by an increase in the carrier concentration. The optical properties and the properties of the electronic bands associated with the defect states of annealed $\mathrm{TiO}_{2-x}$ thin films, e.g., their localization, are reflected in a shift of the absorption to lower frequencies, i.e., to the near IR. The DS shift from the visible to the near infrared in the annealed sample, in which the MIR band disappears by merging into the Drude term, seems to be naturally explained by a progressive delocalization of the electrons with increasing oxygen content, eventually giving a more metallic sample. We therefore demonstrate that, by controlling the growth conditions and/or the UHV annealing process, the light absorption of $\mathrm{TiO}_{2}$ in the UV and visible range can be pushed into the visible and near-IR spectral region.

\section{ACKNOWLEDGMENTS}

This work is performed in the framework of the Nanoscience Foundry and Fine Analysis (NFFA-MIUR Italy Progetti Internazionali) facility. We thank Dr. Goran Drazic of the Institute of Nanochemistry in Ljubljana, Slovenia, for scientific discussions on the HRTEM experiments.

[1] U. Diebold, The surface science of titanium dioxide, Surf. Sci. Rep. 48, 53 (2003).

[2] A. Fujishima, X. Zhang, and D. A. Tryk, $\mathrm{TiO}_{2}$ photocatalysis and related surface phenomena, Surf. Sci. Rep. 63, 515 (2008).

[3] M. A. Henderson, A surface science perspective on $\mathrm{TiO}_{2}$ photocatalysis, Surf. Sci. Rep. 66, 185 (2011).

[4] C. Di Valentin and A. Selloni, Bulk and surface polarons in photoexcited anatase $\mathrm{TiO}_{2}$, J. Phys. Chem. Lett. 2, 2223 (2011). 
[5] K. Hashimoto, H. Irie, and A. Fujishima, $\mathrm{TiO}_{2}$ photocatalysis: A historical overview and future prospects, Jpn. J. Appl. Phys. 44, 8269 (2005).

[6] A. Fujishima and K. Honda, Electrochemical photolysis of water at a semiconductor electrode, Nature 238, 37 (1972).

[7] B. O'Regan and M. Grätzel, A low-cost, high-efficiency solar cell based on dye-sensitized colloidal $\mathrm{TiO}_{2}$ films, Nature 353, 737 (1991).

[8] H. Y. Jeong, J. Y. Lee, and S.-Y. Choi, Interface-engineered amorphous $\mathrm{TiO}_{2}$-Based resistive memory devices, Adv. Funct. Mater. 20, 3912 (2010).

[9] A. Regoutz, I. Gupta, A. Serb, A. Khiat, F. Borgatti, T.-L. Lee, C. Schlueter, P. Torelli, B. Gobaut, M. Light, D. Carta, S. Pearce, G. Panaccione, and T. Prodromakis, Role and optimization of the active oxide layer in $\mathrm{TiO}_{2}$-based RRAM, Adv. Funct. Mater. 26, 507 (2016).

[10] C. Baeumer, C. Funck, A. Locatelli, T. O. Mentes, F. Genuzio T. Heisig, F. Hensling, N. Raab, C. M. Schneider, S. Menzel, R. Waser, and R. Dittmann, In-gap states and band-like transport in memristive devices, Nano. Lett. 19, 54 (2019).

[11] H. Tang, H. Berger, P. E. Schmid, F. Lévy, and G. Burri, Photoluminescence in $\mathrm{TiO}_{2}$ anatase single crystals, Solid State Commun. 87, 847 (1993).

[12] L. Kavan, M. Grätzel, S. E. Gilbert, C. Klemenz, and H. J. Scheel, Electrochemical and photoelectrochemical investigation of single-crystal anatase, J. Am. Chem. Soc. 118, 6716 (1996).

[13] Y. Zhou, C. Chen, N. Wang, Y. Li, and H. Ding, Stable Ti ${ }^{3+}$ self-doped anatase-rutile mixed $\mathrm{TiO}_{2}$ with enhanced visible light utilization and durability, J. Phys. Chem. C 120, 6116 (2016).

[14] F. Huang, A. Yan, and H. Zhao, in Semiconductor Photocatalysis, Materials, Mechanisms and Applications, edited by Wenbin Cao (IntechOpen, 2016).

[15] G. Panaccione et al., Advanced photoelectric effect experiment beamline at elettra: A surface sciencelaboratory coupled with synchrotron radiation, Rev. Sci. Instrum. 80, 043105 (2009).

[16] C. Bigi, P. K. Das, D. Benedetti, F. Salvador, D. Krizmancic, R. Sergo, A. Martin, G. Panaccione, G. Rossi, J. Fujii, and I. Vobornik, Very efficient spin polarization analysis (VESPA): New exchange scattering-based setup for spin-resolved ARPES at APE-NFFA beamline at elettra, J. Synchrotron. Radiat. 24, 750 (2017).

[17] B. Gobaut et al., Role of oxygen deposition pressure in the formation of ti defect states in $\mathrm{TiO}_{2}(001)$ anatase thin films, ACS Appl. Mater. Interfaces 9, 23099 (2017).

[18] D. L. Windt, IMD-software for modeling the optical properties of multilayer films, Comput. Phys. 12, 360 (1998).

[19] M. Sanchez del Rio and R. J. Dejus, XOP v2.4: Recent developments of the X-ray optics software toolkit, SPIE Proc. 8141, 814115 (2011).

[20] P. Orgiani, C. Bigi, P. Kumar Das, J. Fujii, R. Ciancio, B. Gobaut, A. Galdi, C. Sacco, L. Maritato, P. Torelli, G. Panaccione, I. Vobornik, and G. Rossi, Structural and electronic properties of $\mathrm{Bi}_{2} \mathrm{Se}_{2}$ topological insulator thin films grown by pulsed laser deposition, Appl. Phys. Lett. 110, 171601 (2017).
[21] C. Bigi, P. Orgiani, A. Nardi, A. Troglia, J. Fujii, G. Panaccione, I. Vobornik, and G. Rossi, Robustness of topological states in $\mathrm{Bi} 2 \mathrm{Se} 3$ thin film grown by pulsed laser deposition on (001)-oriented $\mathrm{SrTiO}_{3}$ perovskite, Appl. Surf. Science 473, 190- (2019).

[22] R. W. G. Wyckoff, Structure of Crystals (The Chemical Catalog Company, New York, 1931).

[23] P. Orgiani, A. Yu. Petrov, R. Ciancio, A. Galdi, L. Maritato, and B. A. Davidson, Evidence of direct correlation between out-of-plane lattice parameter and metal-insulator transition temperature in oxygen-depleted manganite thin films, Appl. Phys. Lett. 100, 042404 (2012).

[24] M. Kanai, H. Tanaka, and T. Kawai, Origin of metal-insulator transition temperature enhancement in $\mathrm{La}_{0.8} \mathrm{Ba}_{0.2} \mathrm{MnO}_{3}$ thin films as determined by structural analysis, Phys. Rev. B 70, 125109 (2004).

[25] J. Zhang, H. Tanaka, T. Kanki, J.-H. Choi, and T. Kawai, Strain effect and the phase diagram of $\mathrm{La}_{1-x} \mathrm{Ba}_{x} \mathrm{MnO}_{3}$ thin films, Phys. Rev. B 64, 184404 (2001).

[26] P. Orgiani, C. Aruta, R. Ciancio, A. Galdi, and L. Maritato, Enhanced transport properties in $\mathrm{La}_{x} \mathrm{MnO}_{3-\delta}$ thin films epitaxially grown on $\mathrm{SrTiO}_{3}$ substrates: The profound impact of the oxygen content, Appl. Phys. Lett. 95, 013510 (2009).

[27] R. Ciancio, E. Carlino, G. Rossi, C. Aruta, U. Scotti di Uccio, A. Vittadini, and A. Selloni, Magnéli-like phases in epitaxial anatase $\mathrm{TiO}_{2}$ thin films, Phys. Rev. B 86, 104110 (2012).

[28] R. Ciancio, E. Carlino, C. Aruta, D. Maccariello, F. Miletto Granozio, and U. Scotti di di Uccio, Nanostructure of buried interface layers in $\mathrm{TiO}_{2}$ anatase thin films grown on $\mathrm{LaAlO}_{3}$ and $\mathrm{SrTiO}_{3}$ substrates, Nanoscale 4, 91 (2012).

[29] M. Salluzzo, J. C. Cezar, N. B. Brookes, V. Bisogni, G. M. De Luca, C. Richter, S. Thiel, J. Mannhart, M. Huijben, A. Brinkman, G. Rijnders, and G. Ghiringhelli, Orbital Reconstruction and the Two-Dimensional Electron Gas at the $\mathrm{LaAlO}_{3} / \mathrm{SrTiO}_{3}$ Interface, Phys. Rev. Lett. 102, 166804 (2009).

[30] J.-S. Lee, Y. W. Xie, H. K. Sato, C. Bell, Y. Hikita, H. Y. Hwang, and C.-C. Kao, Titanium $d_{x y}$ ferromagnetism at the $\mathrm{LaAlO}_{3} / \mathrm{SrTiO}_{3}$ interface, Nat. Mater. 12, 703 (2013).

[31] J. Gabel, M. Zapf, P. Scheiderer, P. Schütz, L. Dudy, M. Stübinger, C. Schlueter, T.-L. Lee, M. Sing, and R. Claessen, Disentangling specific versus generic doping mechanisms in oxide heterointerfaces, Phys. Rev. B 95, 195109 (2017).

[32] C. Yun-Zhong, N. Pryds, S. Ji-Rong, S. Bao-Gen, and S. Linderoth, High-mobility two-dimensional electron gases at oxide interfaces: Origin and opportunities, Chinese Phys. B 22, 116803 (2013).

[33] A. Koitzsch, J. Ocker, M. Knupfer, M. C. Dekker, K. Dörr, B. Büchner, and P. Hoffmann, In-gap electronic structure of $\mathrm{LaAlO}_{3}-\mathrm{SrTiO}_{3}$ heterointerfaces investigated by soft x-ray spectroscopy, Phys. Rev. B 84, 245121 (2011).

[34] S. Lupi, A. Nucara, A. Perucchi, P. Calvani, M. Ortolani, L. Quaroni, and M. Kiskinova, Performance of SISSI, the infrared beamline of the ELETTRA storage ring, Jour. Opt. Soc. Am. B 24, 959 (2007).

[35] R. J. Gonzales, R. Zallen, and H. Berger, Infrared reflectivity and lattice fundamentals in anatase $\mathrm{TiO}_{2} \mathrm{~s}$, Phys. Rev. B 55, 7014 (1997). 
[36] B. J. Morgan and G. W. Watson, Polaronic trapping of electrons and holes by native defects in anatase $\mathrm{TiO}_{2}$, Phys. Rev. B 80, 233102 (2009).

[37] M. Dou and C. Persson, Comparative study of rutile and anatase $\mathrm{SnO}_{2}$ and $\mathrm{TiO}_{2}$ : Band-edge structures, dielectric functions, and polaron effects, J. Appl. Phys. 113, 083703 (2013).

[38] E. Baldini, Baldini,L. Chiodo, A. Dominguez, M. Palummo, S. Moser, M. Yazdi-Rizi, G. Auböck, B. P. P. Mallett,

H. Berger, A. Magrez, C. Bernhard, M. Grioni, A. Rubio, and M. Chergui, Strongly bound excitons in anatase $\mathrm{TiO}_{2}$ single crystals and nanoparticles, Nature Communication $\mathbf{8}$, 13 (2017).

[39] S. Moser L. Moreschini, J. Jaćimović, O. S. Barišić, H. Berger, A. Magrez, Y. J. Chang, K. S. Kim, A. Bostwick, E. Rotenberg, L. Forró, and M. Grioni, Tunable Polaronic Conduction in Anatase $\mathrm{TiO}_{2}$, Phys. Rev. Lett. 110, 196403 (2013).

[40] S. Moser, S. Fatale, P. Krüger, H. Berger, P. Bugnon, A. Magrez, H. Niwa, J. Miyawaki, Y. Harada, and M. Grioni, Electron-Phonon Coupling in the Bulk of Anatase $\mathrm{TiO}_{2}$ Measured by Resonant Inelastic X-Ray Spectroscopy, Phys. Rev. Lett. 115, 096404 (2015).

[41] C. Chen, J. Avila, E. Frantzeskakis, A. Levy, and M. C. Asensio, Observation of a two-dimensional liquid of Fröhlich polarons at the bare $\mathrm{SrTiO}_{3}$ surface, Nat. Commun. 6, 8585 (2015).

[42] C. Verdi, F. Caruso, and F. Giustino, Origin of the crossover from polarons to Fermi liquids in transition metal oxides, Nature Communication 8, 15769 (2017).

[43] J. M. Riley, F. Caruso, C. Verdi, L. B. Duffy, M. D. Watson, L. Bawden, K. Volckaert, G. van der Laan, T. Hesjedal, M. Hoesch, F. Giustino, and P. D. C. King, Crossover from lattice to plasmonic polarons of a spin-polarised electron gas in ferromagnetic EuO, Nature Communication 9, 2305 (2018).

[44] P. Calvani, M. Capizzi, F. Donato, S. Lupi, P. Maselli, and D. Peschiaroli, Observation of a midinfrared band in $\mathrm{SrTiO}_{3-y}$, Phys. Rev. B 47, 8917 (1993).

[45] P. Calvani, M. Capizzi, S. Lupi, P. Maselli, A. Paolone, and P. Roy, Polaronic optical absorption in electron-doped and hole-doped cuprates, Phys. Rev. B 53, 2756 (1996).

[46] S. Lupi, P. Maselli, M. Capizzi, P. Calvani, P. Giura, and P. Roy, Evolution of a Polaron Band through the Phase Diagram of $\mathrm{Nd}_{2-x} \mathrm{Ce}_{x} \mathrm{CuO}_{4-y}$, Phys. Rev. Lett. 83, 4852 (1999).

[47] S. Lupi, M. Capizzi, P. Calvani, B. Ruzicka, P. Maselli, P. Dore, and A. Paolone, Fano effect in the $a-b$ plane of $\mathrm{Nd}_{1.96} \mathrm{Ce}_{0.04} \mathrm{CuO}_{4+y}$ : Evidence of phonon interaction with a polaronic background, Phys. Rev. B 57, 1248 (1998).
[48] S. L. Cooper, G. A. Thomas, J. Orenstein, D. H. Rapkine, A. J. Millis, S.-W. Cheong, A. S. Cooper, and Z. Fisk, Growth of the optical conductivity in the $\mathrm{Cu}-\mathrm{O}$ planes, Phys. Rev. B 41, 11605(R) (1990).

[49] A. Perucchi, L. Baldassarre, A. Nucara, P. Calvani, C. Adamo, D. G. Schlom, P. Orgiani, L. Maritato, and S. Lupi, Optical properties of $\left(\mathrm{SrMnO}_{3}\right)_{n} /\left(\mathrm{LaMnO}_{3}\right)_{2 n}$ superlattices: An insulator-to-metal transition observed in the absence of disorder, Nano. Lett. 10, 4819 (2010).

[50] D. Emin, Optical properties of large and small polarons and bipolarons, Phys. Rev. B 48, 13691 (1993).

[51] W. S. Choi, H. K. Yoo, and H. Ohta, Polaron transport and thermoelectric behavior in la-doped $\mathrm{SrTiO}_{3}$ thin films with elemental vacancies, Adv. Func. Mater. 25, 799 (2015).

[52] Y. S. Kim, J. Kim, S. J. Moon, W. S. Choi, Y. J. Chang, J.-G. Yoon, J. Yu, J.-S. Chung, and T. W. Noh, Localized electronic states induced by defects and possible origin of ferroelectricity in strontium titanate thin films, Appl. Phys. Lett. 94, 202906 (2009).

[53] M. Dressel and G. Grüner, Electrodynamics of Solids: Optical Properties of Electrons in Matter (Cambridge University Press, New York, 2002).

[54] T. C. Rödel, F. Fortuna, F. Bertran, M. Gabay, M. J. Rozenberg, A. F. Santander-Syro, and P. Le Fevre, Engineering two-dimensional electron gases at the (001) and (101) surfaces of $\mathrm{TiO}_{2}$ anatase using light Phys, Rev. B 92, 041106(R) (2015).

[55] R. Yukawa, M. Minohara, D. Shiga, M. Kitamura, T. Mitsuhashi, M. Kobayashi, K. Horiba, and H. Kumigashira, Control of two-dimensional electronic states at anatase $\mathrm{TiO}_{2}(001)$ surface by $\mathrm{K}$ adsorption, Phys. Rev. B. 97, 165428 (2018).

[56] C. Bigi, Z. Tang, G. M. Pierantozzi, P. Orgiani, P. K. Das, J. Fujii, I. Vobornik, T. Pincelli, A. Troglia, T.-L. Lee, R. Ciancio, G. Drazic, A. Verdini, A. Regoutz, P. D. C. King, D. Biswas, G. Rossi, G. Panaccione, and A. Selloni, Distinct behavior of localized and delocalized carriers in anatase $\mathrm{TiO}_{2}(001)$ during reaction with $\mathrm{O}_{2}$, Phys. Rev. Materials 4, 025801 (2020).

[57] A. F. Santander-Syro, O. Copie, T. Kondo, F. Fortuna, S. Pailhès, R. Weht, X. G. Qiu, F. Bertran, A. Nicolaou, A. Taleb-Ibrahimi, P. Le Févre, G. Herranz, M. Bibes, N. Reyren, Y. Apertet, P. Lecoeur, A. Barthélémy, and M. J. Rozenberg, Two-dimensional electron gas with universal subbands at the surface of $\mathrm{SrTiO}_{3}$, Nature 469, 189 (2011).

[58] Z. Wang et al., Tailoring the nature and strength of electronphonon interactions in the $\mathrm{SrTiO}_{3}(001)$ 2D electron liquid, Nat. Mater. 15, 835 (2016).

[59] J. M. Luttinger, Fermi surface and some simple equilibrium properties of a system of interacting fermions, Physical Review 119, 1153 (1960). 